\title{
Shape coexistence in near Magic shell nucleus ${ }^{52} \mathrm{Cr}$
}

\author{
Rajesh Kumar \\ Dept. of Physics, JCDAV College Dasuya, Punjab (India) \\ *Corresponding Author: rkthakur1@yahoo.co.in, Tel.: +91-8054349781
}

Available online at: www.isroset.org

Received: 07/Apr/2019, Accepted: 26/Apr/2019, Online: 30/Apr/2019

\begin{abstract}
The fp-shell nucleus ${ }^{52} \mathrm{Cr}$ is an even-even nuclei near magic number is with closed neutron shell is at $\mathrm{N}=28$. It was found that coexistence between spherical and deformed shapes can take place rather predominantly in and near semi-magic or doubly magic nuclei. So this nucleus may provide a valuable insight is into the interplay between single-particle and collective modes of excitation. High-spin states in ${ }^{52} \mathrm{Cr}$ have been studied using ${ }^{27} \mathrm{Al}\left({ }^{28} \mathrm{Si}, 3 \mathrm{p}\right){ }^{52} \mathrm{Cr}$ fusion evaporation reaction at beam energy of $70 \mathrm{MeV}$. The level scheme of ${ }^{52} \mathrm{Cr}$ has been extended up to $\mathrm{Ex} \sim 10 \mathrm{MeV}$. Spins and parities have been assigned to many of the new levels on the basis of the directional correlations and linear polarization measurements using clover detectors. The band structures are discussed in the framework of cranked Woods-Saxon and microscopic projected deformed HartreeFock (HF) models. The prolate-oblate shape coexistence is proposed in ${ }^{52} \mathrm{Cr}$ nucleus.
\end{abstract}

Keywords - $\gamma-\gamma$ coincidence, spin-parity, fusion evaporation reaction, Electromagnetic transitions, $\gamma$-transitions and level energy, shapecoexistence

\section{INTRODUCTION}

The spectroscopy of $1 \mathrm{f}_{7 / 2}$ nuclei provides a good test for shell model calculations and associated effective interaction. A great amount of work, from both theoretical and experimental sides, was done in this mass region. Most of the nuclei from ${ }^{40} \mathrm{Ca}$ to ${ }^{56} \mathrm{Ni}$ are well described by a shell model in which the most important configurations are $\left(f_{7 / 2}\right) n$, and $\left(f_{7 / 2}\right) n-r\left(f_{5 / 2} p_{3 / 2} p_{1 / 2}\right) r$, where $(r=1,2, \ldots) \quad[1-3]$. Strong deformation was attributed to nuclei near the middle of the shell, i.e., ${ }^{48} \mathrm{Cr}$ and the neighbouring nuclei. Yrast spectroscopy of the majority of fp-shell nuclei follows the shell model expectation, exhibited somewhat irregular level spacings, often with a marked discontinuity at the termination of the $f_{7 / 2}$ band of states at $J_{\max }=1 / 2[(\mathrm{Z}-20)(28$ $\mathrm{Z})+(\mathrm{N}-20)(28-\mathrm{N})]$. This is particular apparent in the nuclei near $\mathrm{N}$ or $\mathrm{Z}=20,28$ where $\mathrm{J}_{\max }$ is not large. At the middle of shell, near ${ }^{48} \mathrm{Cr}$ for which $\mathrm{J}_{\max }=16$, a different structure appears. However, due to experimental difficulties, only relatively low spin states were identified. In addition, shell model calculations in this region has been mainly restricted to the $f_{7 / 2}$ shell or a few particle excitations in the fp-shell. It is only recently, with the development of sophisticated detection techniques as well as the extension of theoretical calculations, have opened the possibility of studying these relatively light nuclei at high spins where the interplay between single particle and collective degrees of freedom is expected to be clearly exhibited. The nucleus ${ }^{48} \mathrm{Cr}$, with four protons and four neutrons outside the doubly closed shell nucleus ${ }^{40} \mathrm{Ca}$, has the maximum number of particles to develop deformation in the $f 7 / 2$ shell and displays, in fact, a rotational-like ground state band. This band has been recently extended to higher spins $[4,5]$ and the shell model predictions [6] are in very good agreement with the observed level scheme. A complementary comparison with deformed mean field approaches, such as the cranked Hartree Fock Bogoliubov (CHFB) method, allows for a better understanding of the underlying dynamical properties. Similarly Martinez-Pinedo and co-workers [7] have performed theoretical studies for ${ }^{50} \mathrm{Cr}$ in the framework of the full fp spherical shell model and of the CHFB approach, which have predicted two backbending regions in this nucleus. Later the phenomena of backbending has been confirmed experimentally by the work of Lenzi et al.[8] and first backbending was interpreted as a consequence of a change of shape from collective prolate to triaxial or noncollective oblate, due to the gradual alignment of the individual valence particles in the $f_{7 / 2}$ shell. Backbending phenomena, as well as changes of shape, can be understood in terms of the dynamical evolution of the interactions with increasing excitation energy and angular momentum. In $\mathrm{f}_{7 / 2}$ shell nuclei, neutron-proton correlations play a crucial role because valence nucleons are filling the same shell. Therefore, an interpretation of the backbending based on the alignment of like-nucleon pairs, which holds for higher masses where neutrons and protons align independently, has to be revised in this mass region. The rotational properties of 
the $\mathrm{f}_{7 / 2}$ nuclei are also affected by the underlying microscopic structure since the valence nucleons can align fully along the rotational axis creating band terminating states.

The study of high spin states in $\mathrm{N} \sim \mathrm{Z}, \mathrm{f}_{7 / 2}$ nuclei is of current interest, because these nuclei provide an excellent opportunity to study the interplay between single particle and collective degrees of freedom as a function of both angular momentum and number of valence particles. Recent important improvements both in the theoretical and experimental sides have allowed understanding different properties such as collective behaviour, band termination, backbending, spherical-deformed shape coexistence and other related phenomena. The excited states of these fp-shell nuclei, including those with fairly large spins, have been interpreted in terms of pure shell model configurations [912]. Coexistence between spherical and deformed shapes can take place rather predominantly in and near semi-magic or doubly magic nuclei. In general, a closed magic shell leads to a spherical configuration for yrast states, while the coexisting deformed mode is produced by breaking the magic shell. Recently, such a spherical-deformed shape coexistence has been experimentally found in the doubly magic nucleus ${ }_{56} \mathrm{Ni}$ [5] and was also suggested for ${ }_{52} \mathrm{Cr}$ and ${ }_{54} \mathrm{Fe}$ by Mizusaki et al. [6]. The nuclei ${ }^{52} \mathrm{Cr}$ has been studied earlier [13-16] but the band structure has not been properly identified. Thus one of the main motivating factors is to identify the band structure and to look for the coexistence between sphericaldeformed shapes in ${ }^{52} \mathrm{Cr}$ nucleus. The purpose of the present in-beam gamma spectroscopic investigation is to explore structural features of ${ }^{52} \mathrm{Cr}$ nucleus, to resolve the ambiguities regarding the spin and parity assignment for the high spin states. Section I contains the introduction, in section II experimental detail are discussed. The results and discussion part is presented in section III and finally section IV concludes research work with future directions.

\section{EXPERIMENTAL DETAILS}

In the present experiment the ${ }^{52} \mathrm{Cr}$ nucleus was populated using the ${ }^{27} \mathrm{Al}\left({ }^{28} \mathrm{Si}, 3 \mathrm{p}\right){ }^{52} \mathrm{Cr}$ fusion evaporation reaction at beam energy of $70 \mathrm{MeV}$. The beam was provided by the 15UD Pelletron facility at Inter University Accelerator Centre (IUAC), New Delhi India. An isotopically enriched $500 \mu \mathrm{g} / \mathrm{cm}^{2}$ thick ${ }^{27} \mathrm{Al}$ target was used. The de-exciting gamma rays were detected using the Indian National Gamma Array (INGA), at IUAC, New Delhi. For this experiment, the INGA comprised of eight Compton-suppressed Clover detectors combined with heavy Ion Reaction Analyzer (HIRA). A total of about 400 million two- or higher fold $\gamma-$ $\gamma$ coincidence events were collected during the experiment. Efficiency and energy calibration were performed with the standard $\gamma$-ray ${ }^{152} \mathrm{Eu}$ and ${ }^{133} \mathrm{Ba}$ radioactive sources. After gain-matching, the coincidence events were sorted into symmetric and asymmetric (angle dependent) matrices for detailed offline analysis.

\section{RESULTS AND DISCUSSION}

The level scheme of ${ }^{52} \mathrm{Cr}$ resulting from the present work is shown in Fig.1. The transitions and their placement in the level scheme have been determined by $\gamma-\gamma$ coincidences, $\gamma$ ray intensities and sum energy relations of transitions. Spins and parities of the states have been determined from the $R_{D C O}$ ratios and polarization measurements. Several new transitions have been identified in the present work. These transitions are marked by asterisk in the level scheme. The band structure and the level scheme has been extended up to the excitation energy of $10 \mathrm{MeV}$.

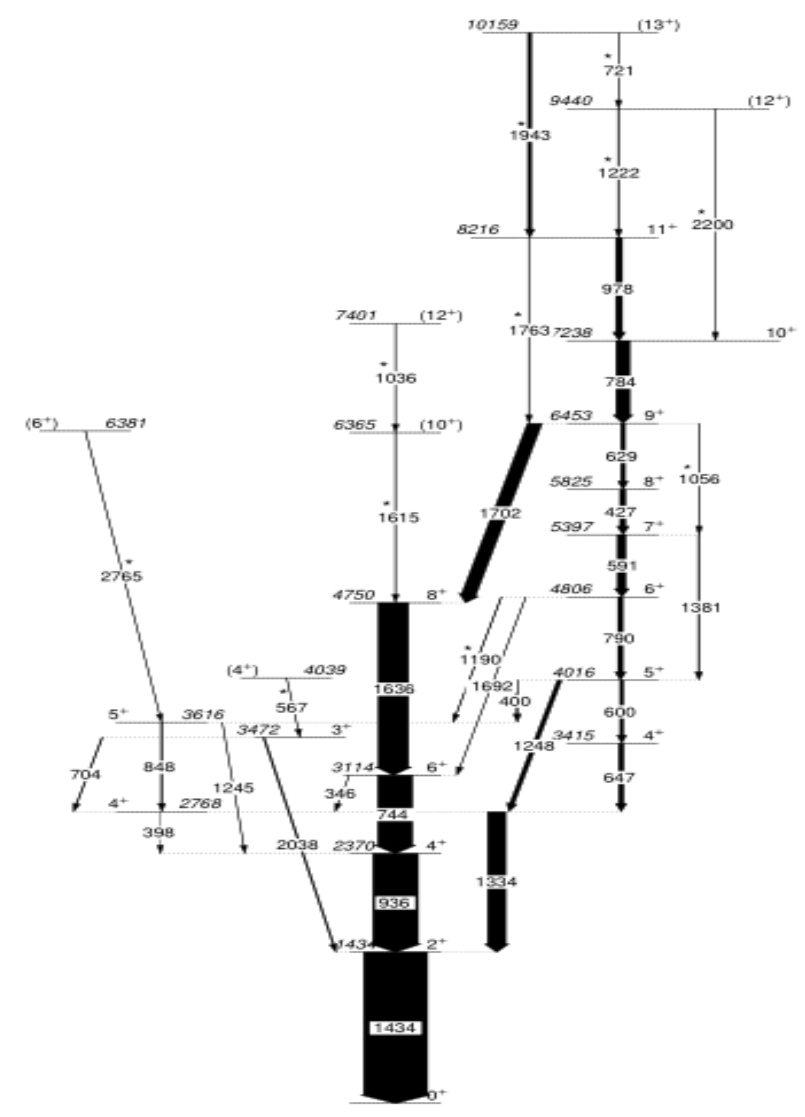

Figure 1: Level scheme for ${ }^{52} \mathrm{Cr}$. The spin and parity assignments, given in parentheses, are tentative. The energies are marked within $\pm 1 \mathrm{keV}$.

The experimental results are compared with the deformed Hartree-Fock model (HF) [17]. Both the oblate and the prolate orbits are considered for $\mathrm{J}$ projection. The $\mathrm{K}=0^{+}$ band is properly understood if, we consider $\mathbf{J}$ projection from oblate orbits only. Among the oblate configurations only $\mathrm{K}=$ $\mathrm{O}^{+}$oblate gives the ground collective band; none of the other possible oblate configurations contribute to collectivity in the spectrum. For the $\mathrm{K}=4^{+}$band we have considered prolate configuration because the collectivity shown by this band is 
only understood by $\mathrm{J}$ projection from prolate HF solu1tion. As seen in Fig. 2, theoretical and experimental spectra are in reasonable good agreement. Thus this nucleus shows the presence of the oblate and prolate shape coexistence.

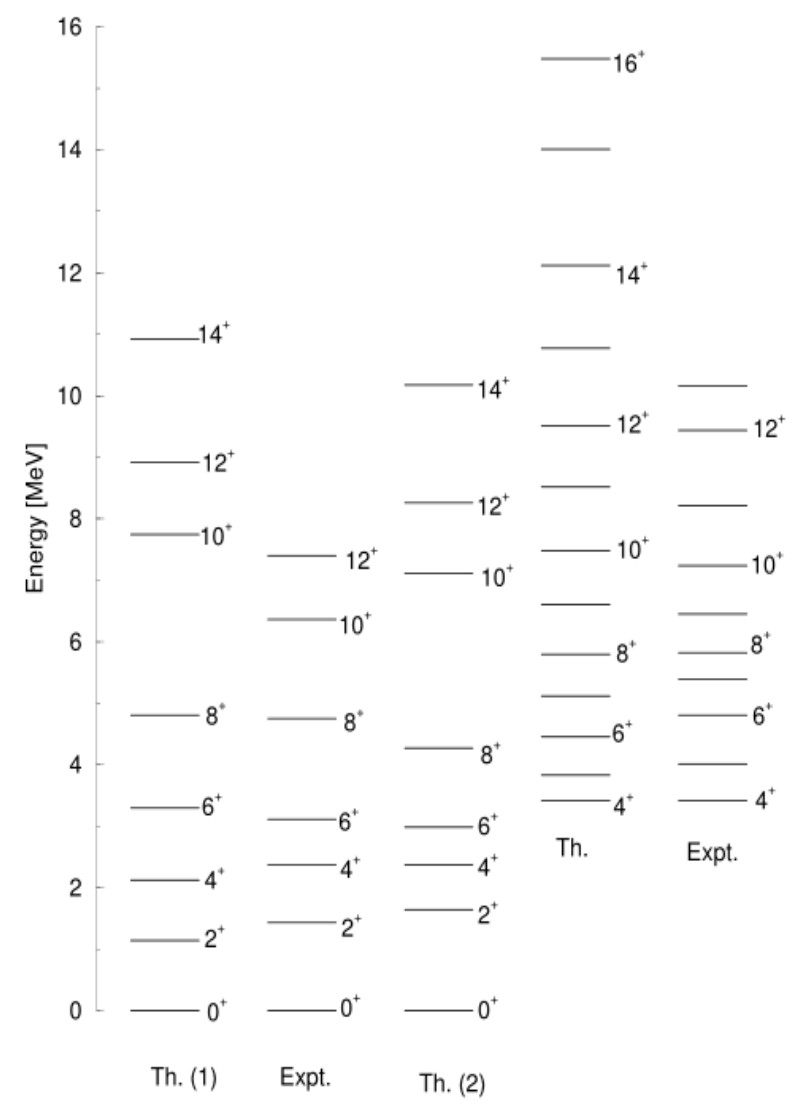

Figure 2: Comparison of experimental levels with the results of deformed microscopic HF model.

To understand the prolate oblate shape coexistence further, the Total Routhian Surface (TRS) calculations have been performed withinWood-Saxon cranking formalism [18, 19] for ${ }^{52} \mathrm{Cr}$. For these calculations the average mean field is taken to be a rotating Wood-Saxon potential [20, 21] with monopole type of pairing interaction. The TRS results are plotted in figure 3 for the frequencies $h \omega=0.0$ and $h \omega=0.55$ $\mathrm{MeV}$ respectively. We have seen that the TRS plot for ground state $(\mathrm{h} \omega=0.0)$ shows a broad minimum at $\gamma=-30^{\circ}$ as shown in figure 3(a). This indicates the oblate structure for the ground state band with negligible deformation consistent with the very small value of experimental $\mathrm{B}(\mathrm{E} 2)=0.0132$ (eb)2 [22,23]. The ground state spectrum is consistent with microscopic Hartree-Fock calculation also indicating a small oblate deformation. At $\mathrm{h} \omega=0.55 \mathrm{MeV}$ a sharp prolate minimum for deformation $\beta_{2} \sim 0.20$ and $\gamma=0^{\circ}$ is formed as shown in figure $3(\mathrm{~b})$, along with a oblate minima with $\beta 2 \sim$ 0.03. The other minima shown in figure $3(\mathrm{~b})$ are of little consequence because of their very poor intensities. This model therefore also indicates the oblate and prolate shape coexistence at higher excitation, consistent with the projected Hartree-Fock microscopic calculations.
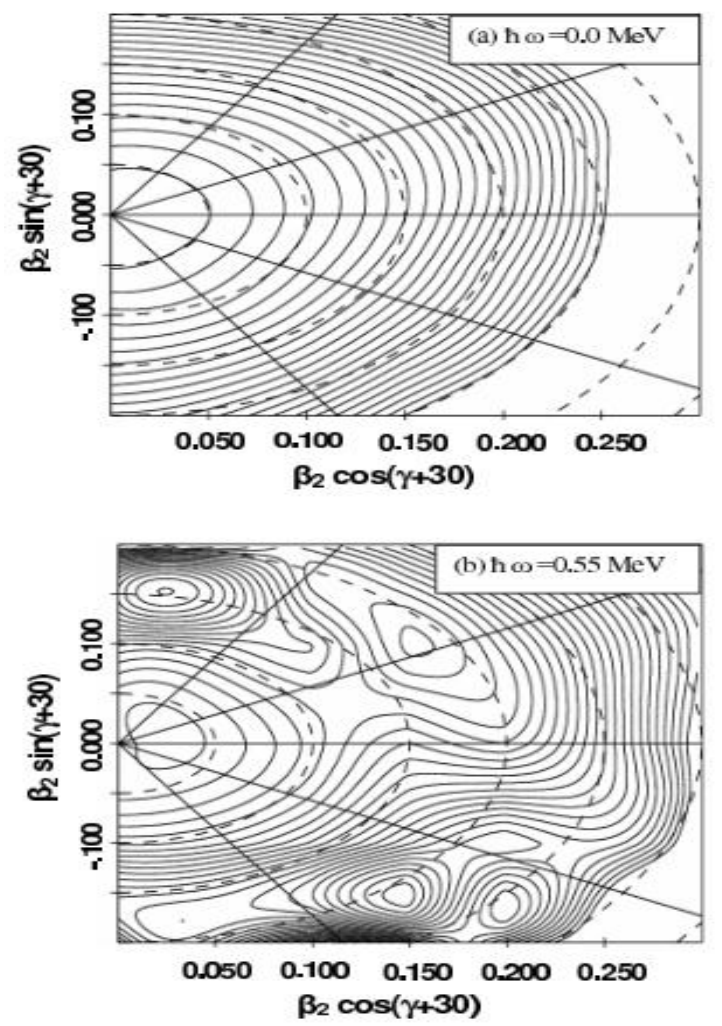

Figure 3: Total Routhian surface plots in $\beta_{2}-\gamma$ plane for the positive parity band in ${ }^{52} \mathrm{Cr}$ for the rotational frequencies $\mathrm{h} \omega=0.0$ $\mathrm{MeV}$, and $\sim \mathrm{h} \omega=0.55 \mathrm{MeV}$ are shown in (a) and (b) respectively.

\section{CONCLUSION}

The high spin states in $\mathrm{N}=28$ even-even ${ }^{52} \mathrm{Cr}$ nucleus have been studied using ${ }^{27} \mathrm{Al}\left({ }^{28} \mathrm{Si}, 3 \mathrm{p}\right){ }^{52} \mathrm{Cr}$ fusion evaporation reaction at beam energy of $70 \mathrm{MeV}$. Several new transitions belonging to this nucleus have been identified extending the level scheme up to excitation energy of $10 \mathrm{MeV}$. Spins and parities have been assigned to many of the new levels on the basis of the directional correlations and linear polarization measurements. The observed level scheme is compared with the microscopic Projected Hartree-Fock calculation and the results are found to be in a reasonably good agreement with theory considering the mixed configuration for the ground band and the prolate configurations for the excited $\mathrm{K}=4^{+}$ band. The cranked Woods-Saxon model also suggest a oblate ground- state band with very small deformation, in agreement with a very small experimental B(E2) value. 


\section{REFERENCES}

[1] W. Kutschera, B. A. Brown, and K. Ogawa, Riv. Nuovo Cimento 1, 12 (1978)

[2] A. Poves and A. Zuker, Phys. Rep. 70, 235 (1981).

[3] R. B. Mooy and P. M. W. Glaudemans, Z. Phys. A312, 59 (1983).

[4] S. M. Lenzi, D. R. Napoli, A. Gadea, M. A. Cardona, D. Hojman, M. A. Nagarajan, C. Rossi Alvarez, N. H. Medina, G. de Angelis, D. Bazzacco, M. E. Debray, M. De Poli, S. Lunardi, D. de Acuna, Z. Phys. A354, 117 (1996).

[5] J. A. Cameron, J. Jonkman, C. E. Svensson, M. Gupta, G. Hackman, D. Hyde, S. M. Mullins, J. L. Rodriguez, J. C. Waddington, A. Galindo-Uribarri, H. R. And rews, G. C. Ball, V. P. Janzen, D. C. Radford, D. Ward, T. E. Drake, et al., Phys. Lett. B387, 266 (1996).

[6] E. Caurier, J. L. Egido, G. Martinez-Pinedo, A. Poves, J. Retamosa, L. M. Robledo, et al., Phys. Rev. Lett. 75, 2466 (1995).

[7] G. Martinez-Pinedo, A. Poves, L. M. Robledo, E. Caurier, F. Nowacki, J. Retamosa, and A. Zuker, Phys. Rev. C54, R2150 (1996).

[8] S. M. Lenzi, C. A. Ur, D. R. Napoli, M. A. Nagarajan, D. Bazzaco, D. M. Brink,

[9] K. Lips and M. T. McEllistrem, Phys. Rev. C1, 1009 (1970).

[10] I. P. Johnstone and H. G. Benson, J. Phys. G3, L69 (1977).

[11] A. Yokoyama, T. Oda, and H. Horie, Prog. theor. Phys. 60, 427 (1978).

[12] I. P. Johnstone, Phys. Rev. C17, 1428 (1978).

[13] S. W. Sprague, R. G. Arns et al., Phys. Rev. C4, 2074 (1971).

[14] A. Berinde et al., Nucl. Phys. A284, 65 (1977).

[15] D. Evers, A. Harasim, R. L. McGrath, and W. Assmann, Phys. Rev. C63, 1690 (1977).

[16] P. Banerjee, B. Sethi, J. M. Chatterjee et al., Phys. Rev. C36, 2274 (1987).

[17] G. Ripka, Advances in Nuclear Physics,Vol. 1 (1966) Ed.M.Baranger and .Vogt (Plenum).

[18] P. Ring and P. Schuck, The Nuclear Many Body Problems (SpringerVerlag, Berlin, 1980), p. 244.

[19] W. Nazarewicz, M. A. Riley, and J. D. Garrett, Nucl. Phys. A512, 61 (1990).

[20] R. Ernst et al., Phys. Rev. Lett. 84, 416 (2000).

[21] C. W. Towsley et al., Nucl. Phys. A250, 381 (1975).

\section{AUTHORS PROFILE}

Dr. Rajesh Kumar is Ph.D. in experimental Nuclear Physics (Physical Science) from Panjab Univeristy, Chandigarh 2008. He is currently working as Asstt. Professor in Department of Physics, PG Department of Physics, JCDAV College (Affiliated to Panjab University) since 2009. He has published more than 25 research papers in reputed international journals including Thomson Reuters (SCI \& Web of Science), symposia and conferences. The main research work focuses in nuclear structure. He has 12 years of teaching experience and 16 years of research experience. 